\title{
Cholera in Yemen: concerns remain over recent spike but control efforts show promise
}

Ahmed Al-Mandhari, ${ }^{1}$ Altaf Musani, ${ }^{2}$ Abdinasir Abubakar ${ }^{3}$ and Mamunur Malik ${ }^{4}$

\begin{abstract}
${ }^{1}$ Regional Director, World Health Organization Regional Office for the Eastern Mediterranean, Cairo, Egypt. ${ }^{2}$ WHO Country Representative, Sana'a, Yemen. 3Infectious Hazard Management, Department of Health Emergency, World Health Organization Regional Office for the Eastern Mediterranean, Cairo, Egypt. "Infectious Hazard Management, World Health Organization Regional Office for the Eastern Mediterranean, Cairo, Egypt. (Correspondence to: Mamunur Malik: malikm@who.int).
\end{abstract}

Citation: Al-Mandhari A; Musani A; Abubakar A; Malik MR. Cholera in Yemen: concerns remain over recent spike but control efforts show promise. East Mediterr Health J. 2018;24(10):971-972. https://doi.org/10.26719/2018.24.10.971

Copyright @ World Health Organization (WHO) 2018. Some rights reserved. This work is available under the CC BY-NC-SA 3.0 IGO license (https:// creativecommons.org/licenses/by-nc-sa/3.0/igo).

October 2018 would be two years since the world came to know of the first case of cholera in war-torn Yemen. Soon after, the outbreak developed at an unprecedented scale and turned into one of the worst historical cholera events in terms of cases reported (1). By the end of September 2018, more than one million suspected cases had been reported across the country in all but one governorate (2), where approximately $60 \%$ of these cases remained concentrated in roughly half of the 330 districts in the country (3).

How many of these million cases are "true" cholera could not be ascertained due to inadequate laboratory testing capacity in Yemen. Despite this uncertainty, such high number of cases with rapid geographic spread, raging over such a long period and showing little signs of waning, has already raised alarm. Although the outbreak started in October 2016, the major upsurge of the new cases was seen after May 2017 and the country continued to report a high case load until early 2018. Recently, the surveillance data shows a declining trend when compared to the same period last year. The number of suspected cases has ranged from about 1500 to 5000 per week-a drop from 2017, when approximately 5000 new cases were reported every day during the same period in 2017 (4). Not only have fewer cases been reported in recent months, but also fewer districts have reported suspected cholera cases compared to the same period in 2018. However, the recent spike in reported cholera case numbers continues to be worrisome.

Cholera is one of the oldest foes to humanity. Humanitarian crisis resulting from war or any other protracted and complex emergencies resulting in collapse or near collapse of health systems provide an ideal environment for emergence and re-emergence of cholera epidemics, as witnessed in Haiti, Zimbabwe and now in Yemen (3). Despite being one of the oldest killer diseases the world continues to face, the technical solutions to control and even eliminate the risk of cholera are easily available, affordable and feasible to implement. The disease can be easily treated; for over $80 \%$ of cases, simple rehydration therapy means in just a few hours the patient can walk home from health facilities after receiving treatment, provided the access to healthcare early is unimpeded.
A recent meeting organized by the World Health Organization (WHO) and United Nations International Children's Emergency Fund (UNICEF) in Amman, Jordan, revisited the current operational strategies to control cholera in Yemen. Following a recent upsurge in reported cholera cases, a set of potentially high-value and highimpact strategies were considered to accelerate control efforts for an immediate halt to the progression of the epidemic, after showing signs that transmission is likely slowing down.

First, the current evolution of cholera surveillance data would be studied more in-depth in order to identify 1) districts with active transmission; 2) districts where transmission shows signs of waning; and 3) districts where the transmission probably has stopped. The control efforts would be scaled up to target districts with active transmission with the goal of achieving "zero transmission" within the next three to six months.

Second, currently over 600 ooo people living in the disease hotspot districts have been protected from cholera through a mass immunization campaign with two-dose inactivated oral cholera vaccines (OCVs) between May and September 2018. The country received 1.3 million doses of OCVs from the Global task Force on Cholera Control (GTFCC) for conducting pre-emptive vaccination campaign in high-risk districts. The remaining doses currently available in the country would have to be used to judiciously target districts where active transmission is ongoing in order to achieve "zero" transmission. Despite earlier apprehension that mass immunization campaign with a two-dose OCV (administered 10-14 days apart) would be logistically difficult in the context of Yemen due to security issues and mobile populations, the success of protecting over half a million people preemptively against a rapidly spreading outbreak will go a long way in halting the epidemic. Experience has already demonstrated that deployment of OCV, reactively, in epidemics can also be effective (4-6).

Third, since cholera is a water-borne disease that thrives in poor water and sanitation situations and transmission is fecal-oral, the only way we can interrupt the transmission is to improve access to clean water. It is plausible that the water and sanitation situation in 
Yemen has near collapsed owing to the protracted crisis and long-term investment would be needed to restore the water and sanitation infrastructure. Providing clean water to nearly 16 million Yemenis who currently lack access to safe water would be a daunting task (4). As transmission needs to be stopped at the household level, it is here that efforts should now be targeted in those disease hotspots showing continued transmission. A variety of interventions should be considered to scale up mass distribution of chlorinated water or chlorination/ water purification tablets, provide hygiene kits with soap for hand washing, and provide training on making water safe for drinking and safe disposal of human waste at the household level. An active collaboration and engagement between the health and water and sanitation / hygiene (WASH) sector will be critical to achieve this at the household level. The cholera surveillance data should guide these interventions and prioritize areas to target.

Fourth, all cases, including those mild cases not seeking healthcare owing to lack of access or healthseeking behaviour, should be brought under the cover of treatment through community-based outreach services or establishing oral rehydration therapy (ORT) centres at community level. Cholera transmission can only be interrupted if we can interrupt its transmission through treating all dehydrated cases, since this would minimize repeated purging of Vibrio bacterium in the environment and limit its propagation.

The meeting also underscored the need to understand better the transmission pathways of such a large scale epidemic with a low fatality case rate. However, it is unclear what have been the drivers of the noted cholera spike as well as the prolonged and sustained cholera transmission in Yemen, including the epidemiological and environmental determinants. A number of other knowledge gaps were also identified in the meeting that require active collaboration between Yemeni health authorities, WHO, UNICEF, international organizations and academia.

Understanding the full genome sequence of the Vibrio bacterium to detect if the circulating strain has mutated into a more virulent or toxigenic strain, along with better predictive models and real-time analysis of risk factors for deaths, were some of the key knowledge gaps that require more evidence in order to accelerate cholera response. The meeting also looked at the current strategy for identifying and reporting suspected cases, adherence of healthcare workers to case definition, and current strategy for laboratory testing.

Records indicate that the last outbreak of cholera was officially reported from Yemen in 2011 (7). The unprecedented scale of this current outbreak has exceeded all our previous understandings of the disease's duration and transmission patterns. However, after two years of continued efforts to halt the interruption, we clearly see positive signs of ending cholera. International agencies including WHO and UNICEF need to combine and consolidate ongoing efforts in order to overcome remaining obstacles. Recent gains need to be sustained and integrated into a comprehensive long-term plan in line with the "End Cholera - A Road Map for 2030", which WHO Member States endorsed during the Seventy-First World Health Assembly in 2018, and vowed to reduce by $90 \%$ the magnitude of cholera outbreaks with the support of Global Task Force on Cholera Control (GTFCC) partners.

It is hoped that this collective experience, knowledge and partnership in controlling cholera in one of the worst humanitarian crisis settings ever seen, also be used to control other infectious disease threats in the country, and help recover and improve the public health system in Yemen.

\section{References}

1. Von Seidlein L, Sack D, Azman AS, Ivers LC, Lopez AL, Deen JL. Cholera outbreak in Yemen. Lancet Gastroenterol Hepatol. 2017 Nov;777:(11)2. https://doi.org/10.1016/S-30287(17)1253-2468X PMID: 29017710

2. Al-Mekhlafi HM. Yemen in a Time of Cholera: Current situation and challenges. Am J Trop Med Hyg. 2018 Jun;1562-1558:(6)98. https://doi.org/10.4269/ajtmh.0811-17 PMID: 29557331

3. Camacho A, Bouhenia M, Alyusfi R, Alkohlani A, Naji MAM, de Radigues X, et al. Cholera epidemic in Yemen, 18-2016: an analysis of surveillance data. Lancet Glob Health. 2018 Jun;6)6):e-680e69o https://doi.org/10.1016/S109-2214X(4-30230(18 PMID: 29731398

4. Qadri F, Islam T, Clemens JD. Cholera in Yemen - An old foe rearing its ugly head. N Engl J Med. 7-2005:(21)377;2017. https://doi. org/10.1056/NEJMp1712099 PMID: 29091747

5. Ciglenecki I, Azman AS, Jamet C, Serafini M, Luquero FJ, Cabrol J-C. Progress and challenges in using oral cholera vaccines to control outbreaks: The Médecins Sans Frontières experience. J Infect Dis. 2018 Oct 218;15(suppl_3):S-165S166. https://doi. org/10.1093/infdis/jiy487 PMID: 30239901

6. Azman AS, Parker LA, Rumunu J, Tadesse F, Grandesso F, Deng LL, et al. Effectiveness of one dose of oral cholera vaccine in response to an outbreak: a case-cohort study. Lancet Glob Health. 2016 Nov;11)4):e-856e863 https://doi.org/10.1016/S-2214 $109 \mathrm{X}(-30211(16 \mathrm{X}$

7. Cholera, 2011. Weekly epidemiological record / Releve epidemiologique hebdomadaire. 304-289:(32/31)87;2012. (http://www.who. int/wer/2012/wer32_8731.pdf?ua=1). 